\title{
Comparison of Fixation of supracondylar humeral fractures in children by lateral cross-wiring technique versus traditional lateral pinning
}

Abdelrahman A. Sadek, Moustafa Elsayed, Hossam El-Din M. El-Azalb, Hassaan H. Noamany.

\begin{abstract} nerve injury. radiological results.

\section{Introduction}

Supracondylar fractures of the humerus in children accounts for $60 \%$ of all fractures around the elbow. (1) It represents $4-6.5 \%$ of all paediatric fractures. In treatment of non-displaced Type I fractures simple immobilization with a posterior splint applied at 60 $90^{\circ}$ of elbow flexion is preferred. (2) Currently, the treatment of choice for type II fractures is operative reduction and pinning rather than cast. (3) Most cases of type III fractures require operative reduction and pinning. The results of type III fractures treated with closed reduction and cast immobilization are not as good as the results of pinning. (4) There are various options for the pattern of $\mathrm{K}$ wire fixation of displaced supracondylar fractures. Studies found the greatest resistance to rotation occurred with medial-lateral cross pinning. (5) The second most stable pattern was fixation utilizing three lateral diverging pins. The least stable was fixation with two lateral pins, which cross at the fracture site. While medial-lateral cross pinning has the greatest resistance; the disadvantage is the risk of ulnar nerve injury. (6) Lateral pinning is recommended (7) to avoid iatrogenic ulnar nerve injury that
\end{abstract}

Introduction The currently accepted treatment for displaced supracondylar humeral fractures in children is closed reduction and fixation with percutaneous Kirschner wires. The aim of this study was to study the results of a cross-wiring technique, achieved solely from the lateral side, in an effort to reduce the risk of ulnar

Conclusion there was no significant difference between lateral cross-wiring technique and traditional lateral pinning as regard postoperative clinical results and

can occur with medial lateral cross pinning. Although iatrogenic ulnar nerve injuries usually resolve, several permanent iatrogenic ulnar nerve injuries have been described. (8) Closed reduction and lateral crosswiring technique with ascending and descending $\mathrm{K}$-wires is an effective method to treat type II and III supracondylar fractures in children. Regardless of stability, this method can be used to avoid iatrogenic ulnar nerve injuries. (9)

\section{Aim of the work}

The aim of this work is to assess clinical results of fixation of supracondylar humeral fractures by lateral cross-pinning versus traditional lateral pinning in children.

\section{Patients and Methods}

It is a prospective study of 40 children, presented by early displaced supracondylar fracture (Gartland type II \& III) admitted to Orthopaedic Department Sohag University Hospital between June 2015 and January 2016 after approval from the institute ethical committee. Children with previous fracture in the same side, polytraumatized or associated neurovascular injury have been excluded from the study. 
Pre-operative assessment was done at emergency room by resident doctors by taking history from the parents, general evaluation of the general condition, associated other injuries, clinical examination of the fracture side, neurovascular assessment and then radiological examination by antero-posterior and lateral $\mathrm{x}$-rays. Then all children splinted by above elbow slab in $70^{\circ}-90^{\circ}$ of flexion. The operation was done within the first 24 hours after admission. Pre-operative investigations (blood picture and prothrombine time \&concentration) were done for all cases.

Surgical Technique: General anesthesia was used for all patients with the injured upper limb at the side of the table. The injured elbow was placed on the plate of image intensifier which was adequate for the surgery due to the small size of the elbow.
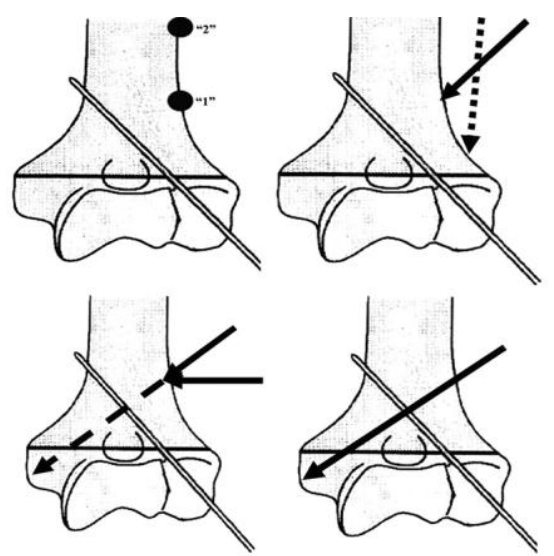

Fig.1 (lateral cross-wiring technique)

a) The point of entry should be in the metaphyseal part ' 1 ' and not in the diaphyseal part ' 2 ' of the humerus.

b) The second wire may skid down the lateral cortex during introduction.

c) The wire is directed at right angles to the cortex until it has penetrated, and then pulled back, and the trajectory is readjusted.

d) The second wire is introduced through the lateral cortex, proximal to the fracture line, and is driven across the fracture into the medial condyle. Wires must cross above the fracture line

For the crossed lateral fixation technique, Two K-wires were used to stabilize the fracture. These wires were passed under $\mathrm{C}$-arm guidance with the elbow held in a hyper flexed position to maintain the initial reduction. The first wire was introduced through the lateral condyle in a retrograde direction (ascending) across the fracture and into the medial cortex. The second wire was introduced through the lateral cortex, proximal to the fracture line, and driven in an ante grade direction (descending) across the fracture line into the medial condyle. The medial condyle should not be penetrated. The wires must cross above the fracture line. Occasionally, the second wire may skid down the lateral cortex during introduction. To avoid this, the wire was directed perpendicular to the cortex until the cortex was penetrated, pulled back and then redirected towards the medial condyle.

For the traditional lateral fixation technique two pins were inserted from lateral aspect of elbow across the lateral cortex to engage the medial cortex under $\mathrm{C}$-arm guidance with the elbow held in a hyper flexed position to maintain the initial reduction. Pins were placed must not cross at fracture site. insertion point is in the center of lateral condyle (capitellum). generally, the pin is aimed $35^{\circ}$ upward and $10^{\circ}$ posterior.

After fixation, the fracture stability was tested under $\mathrm{C}$-arm. The wires were then bent, cut and left outside the skin, facilitating their removal in follow up.

Clinical evaluation was done which includes passive range of motion, measurement of carrying angle, neurovascular status, superficial and deep infection and necessity to reoperate. Clinical evaluation was graded according to carrying angle and elbow range of motion using the criteria of Flynn et.al. (10) Radiographic evaluation was performed by 
anteroposterior and lateral radiographs of the elbow. Follow up radiographs were taken at four weeks, eight weeks, twenty four weeks. Baumann angle and Humero-capitellar angle were calculated on the immediate radiographs and after three months for any difference of Baumann angle and Humero-capitellar angle.

For each child, the carrying angle, ROM, Baumann's angle, capitellohumeral angle and metaphyseal diaphyseal angle measured on both the fractured and normal side to detect the difference between normal and fractured side in each angle.

Criteria of Flynn: Flynn criteria are obtained by measuring the range of elbow movement and the carrying angle. Both loss in carrying angle and loss in elbow motion compared with normal side are scored as follows: between $0^{\circ}$ and $5^{\circ}$ excellent; 6-10 good; $11-15^{\circ}$, fair; more than $15^{\circ}$, poor. ${ }^{(10)}$

\section{Results}

During this study period 40 children were treated for type II and type III supracondylar fracture in humerus. children divided randomly into two groups, group A and B. the mean follow-up period was 27 weeks (range $23-29$ weeks) Group A comprised twenty patients fixed by lateral cross-wiring technique. The mean age was 6.54 years. Among which 15 patients $(75 \%)$ were boys and 5 patients $(25 \%)$ were girls. In 13 patients injury occurred due to fall from height, 6 patients were injured while playing whereas 1 due to road traffic accident. 6 patients $(30 \%)$ had right elbow and $14(70 \%)$ had left elbow fracture. Displacement was posteromedially in 10 patients, 4 had posterolaterally and 6 had direct posterior displacement.

The group-B comprised twenty patients. The mean age was 5.88 years. Among which 14 patients (70\%) were boys and 6 patients (30\%) were girls. In 11 patients injury occurred due to fall from height, 7 patients were injured while playing whereas 2 due to road traffic accident. 9 patients $(45 \%)$ had right elbow and $11(55 \%)$ had left elbow fracture. Displacement was posteromedially in 12 patients, 4 had posterolaterally and 4 had direct posterior displacement.

Clinical results: at the final follow-up were evaluated according to Flynn's grading system ${ }^{(10)}$; According to carrying angle, in group A, results were 11 patients excellent (55\%), six good (30\%), two fair (10\%) and only one poor $(5 \%)$. In group B, 8 were excellent $(40 \%), 7 \operatorname{good}(35 \%)$, three fair $(15 \%)$ and two poor $(10 \%)$. There was no statistically significant difference between the two groups $(p>0.05)$. 
According to functional results, in group A, there were $11(55 \%)$ excellent, 5 $(25 \%)$ good, three $(15 \%)$ fair and only one $(8 \%)$ poor. In group B, there were nine (45\%) excellent, $5(25 \%)$ good, $4(20 \%)$ fair and two (10\%) poor. There was no statistically significant difference between the two groups $(p>0.05)$. The average of loss in carrying angle and range of motion (both flexion and extension) is higher in group B than group A but the difference is statistically not significant.

Radiological results: The difference between means of Baumann's angle, metaphyseal-diaphyseal angle and humero-capitellar angle was compared; p-value found to be more than 0.05 indicating non significant difference.

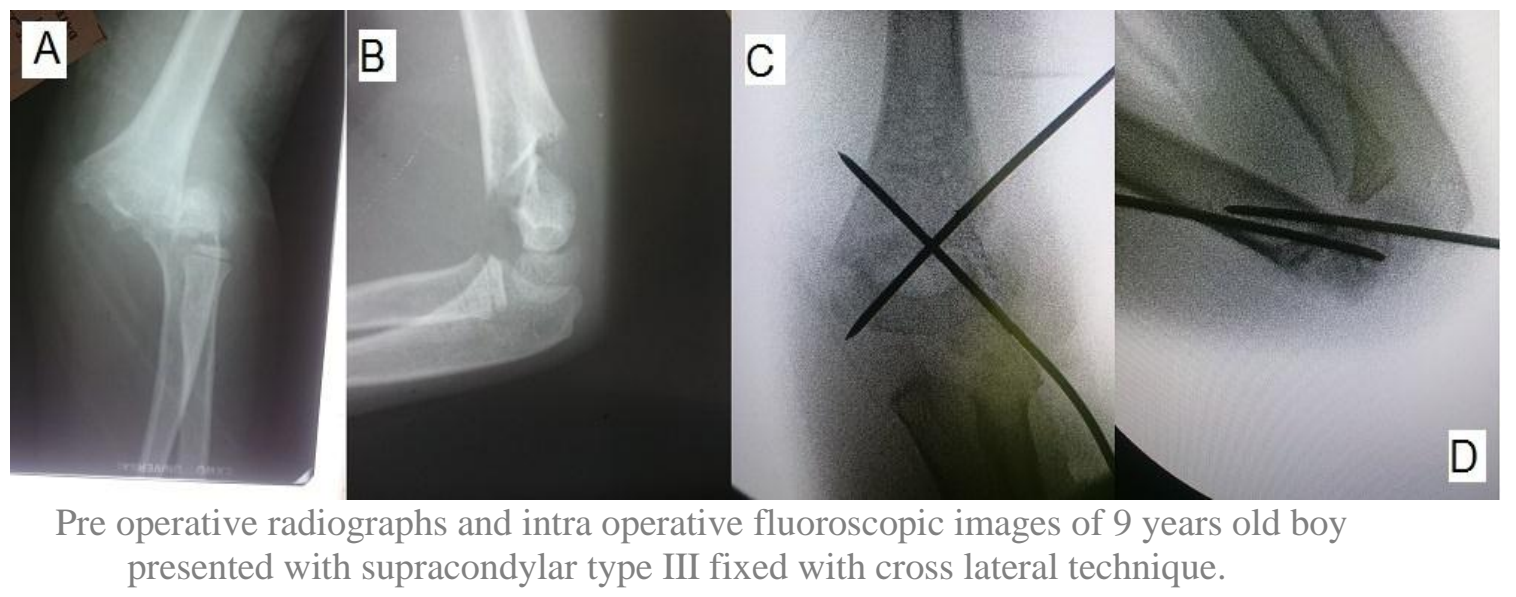

Post-operative complications: 3 patients in group A and 2 in group B had superficial pin site infections which were treated with a course of oral antibiotics with no need for premature removal of the wire in any case. No re-operation was needed in all cases. No neurovascular complications occur in any case.

\section{Discussion}

Supracondylar fracture of the humerus in children is one of the common fractures seen in orthopaedic outpatient department all over the world accounting for $60 \%$ of all elbow fracture in children in the first decade of life. ${ }^{(1)}$

The treatment of supracondylar fractures aims to restore anatomical or near anatomical reduction, early restoring elbow function with good ROM, avoid complications like neurovascular, deformity, elbow stiffness...etc. Decrease physical and psychological impact of the fracture on the children and their parents. ${ }^{(11)}$

Although closed reduction and percutaneous K-wire pinning is the currently accepted treatment of displaced supracondylar fractures of the humerus in children, there is still

argument on the optimal configuration of these K-wires regarding the fracture stability and ulnar nerve safety. ${ }^{(12)}$

This prospective study aims to assess and compare between lateral crosspinning versus traditional lateral pinning as regard stability, safety, complication, postoperative ROM and restoration of function.

This prospective study included 40 children. They were divided randomly into two groups .group A lateral crosswiring technique. Group B traditional lateral pinning, 20 children in each group. The average period of follow up was 27 weeks. Clinically the results evaluated according to criteria of Flynn, The clinical and radiological results between two groups was compared statistically by SPSS program using t- 
test, the differences between two groups is statistically not-significant.

Radial nerve may be at risk with crossed lateral pinning, a case was reported by Gangadharan S. et al. of iatrogenic radial nerve palsy following lateral cross-pinning, a cadaveric study was done to define a safe entry point for the proximal lateral K-wire. A child's cadaveric humerus was pinned laterally in three coronal planes, simulating the proximal entry pin. The radial nerve lay farthest from the wire in the posterolateral plane, 1 and $2 \mathrm{~cm}$ proximal to the lateral epicondyle. Gangadharan S. et al. report the first incidence of radial nerve injury with lateral cross-pinning and suggest that the wire should be placed posterolaterally within $2 \mathrm{~cm}$ from the lateral epicondyle. ${ }^{(13)}$

Queally JM et al. ${ }^{(14)}$ retrospectively reviewed all children who had undergone this procedure over a 10year period. 43 patients, who underwent lateral cross-wiring for displaced supracondylar fractures (Gartland type II and type III) of the humerus were reviewed with a mean follow-up time of 36 months. No major loss of reduction occurred. The mean change in Baumann's angle $\left(4.2^{\circ} \pm 1.6\right)$ between intra-operative and follow-up radiographs was not significant ( $>>0.05$ ). No iatrogenic case of ulnar nerve injury occurred. The 'carrying angle' and 'return to function' in all children had returned to normal relative to the other side. Postoperative complications consisted of three patients developing pin-site infections, which were successfully treated. This study concluded that lateral crosswiring technique is an effective option in treating displaced supracondylar fractures of the humerus in children. It is as effective as the traditional crosswire technique in terms of fracture healing with a reduced risk of ulnar nerve injury.

In the 20 cases of Shannon et al. ${ }^{(15)}$, all children had a full range of elbow motion compared with their normal side, and the mean carrying angle of the injured elbow was $15^{\circ}$ (range, $10-20^{\circ}$ ). There were no intraoperative complications, including ulnar nerve injuries. All complications were related to K-wires.

Another similar series from Eberhardt et al. ${ }^{(12)}$ achieved $93 \%$ good to excellent functional results. Their cosmetic results were $93 \%$ excellent and $7 \%$ good, with no poor results according to Flynn criteria. Radiologically, $87 \%$ of their cases had a normal humeral shaft condylar angle. There was no case of secondary displacement.

Stability studies had demonstrated that crossed pins provides the best stability. Using an adult human cadaver model, Zionts et al. ${ }^{(5)}$ measured the resistance to rotation of the distal fragment of simulated supracondylar fractures fixed with four different pin configurations. They found that the crossed-wire configuration, placed from the medial and the lateral condyles, was the most stable arrangement. They promoted the use of the crossed-pin configuration, but mentioned that with significant swelling, the two lateral parallel pins could be considered as an inferior but acceptable option. More recently, Lee et al. ${ }^{(16)}$ using a saw-bone model, found that two 'divergent' lateral pins were comparable to crosswires in extension, varus and valgus loading, but were inferior in axial rotation testing.

Although Lateral cross-wiring technique does not include supporting biomechanical data, the crossed-wire configuration obtained by inserting both wires from the lateral side is similar to 
that obtained by the traditional medial and lateral technique. ${ }^{(12 ; 15)}$

Sudeep et al. (17) reported a prospective study of 66 children to evaluate the difference between fixation by traditional medial-lateral pinning and traditional lateral pinning. 6 were lost for follow up with mean period of follow up 6 months. No major loss of reduction was observed in both the groups where as there was no significant difference change in Baumann angle, change in Humerocapitellar angle, Flynn grade, elbow extention and flexion, carrying angle, total range of motion. This study concluded that no significant difference between the two methods as regard stability but lateral wires safer as regard iatrogenic ulnar nerve injury.

An important point was the possible difficulty of introduction and oblique direction of the proximal lateral pin. However, truly, this is not so difficult. The periosteum is typically thick in this age group, and so, even with an oblique approach, the pin easily enters the cortex and can then be advanced toward the medial condyle as it traverses the fracture site ${ }^{(12)}$.

There might be a theoretical risk that the proximal lateral pin can injure the ulnar nerve when drilling through the bony area of the medial condyle. The descending pin should not perforate the medial condyle to avoid ulnar nerve injury. This could be verified by fluoroscopy. Regarding this point, like all exclusive lateral-pinning techniques, it is advantageous over the traditional medial/lateral cross-wiring, which had a reported incidence of iatrogenic ulnar nerve injury ranging from 2 to $8 \%{ }^{(18 ;}$ 19)

Another potential complication exclusive to cross-lateral technique is the risk of radial nerve injury at the site of the proximal wire entry. However, this pin does not appear to produce an increased risk to the radial nerve. There is a distance of greater than $2 \mathrm{~cm}$ between the radial nerve in this area on the lateral side and the pin introduction point, provided that the entry is metaphyseal and not higher in the diaphysis. Also, at this level, the radial nerve is anterior to the lateral intermuscular septum. It can be avoided by entering the skin slightly posterior to the mid-coronal plane. ${ }^{(20)}$

\section{Conclusion}

From this prospective study we concluded that there was no significant difference between lateral cross-wiring technique and traditional lateral pinning as regard postoperative clinical results and radiological results. Lateral crosswiring technique may be more stable, but the difference in final clinical results is not significant and crosslateral technique is slightly more difficult and can cause theoretically iatrogenic radial nerve injury.

\section{References}

1. Noaman HH, M.D. Microsurgical reconstruction of brachial artery injuries in displased supracondylar fracture humerus in children. Microsurgery. 2006, 26, pp. 498-505.

2. J., Charnley. Closed Treatment of Common Fractures. Edinburgh: Churchill Livingstone. 1961, pp. 105-115.

3. Battaglia TC, Armstrong DG, Schwend RM. Factors affecting forearm compartment pressures in children with supracondylar fractures of the humerus. $J$ Pediatr Orthop. 2002, 22 (4), pp. 431-439.

4. Cramer KE, Devito DP, Green NE. Comparison of closed reduction and percutaneous pinning versus open reduction and percutaneous pinning in displaced supracondylar fractures of the humerus in children. $J$ Orthop Trauma. 1992, 6(4), pp. 407-412.

5. Zionts LE, McKellop HA, Hathaway. Torsional strength of pin configurations used to fix supracondylar fractures of the 
humerus in children. J Bone Joint Surg Am. 1994, 76, pp. 253-256.

6. Lyons JP, Ashley E, Hoffer MM. Ulnar nerve palsies after percutaneous crosspinning of supracondylar fractures in children's elbows. J Pediatr Orthop. 1998, 18, pp. 43-45.

7. Ariño VL, Lluch EE, Ramirez AM, et al. Percutaneous fixation of supracondylar fractures of the humerus in children. $J$ Bone Joint Surg Am. 1977, 59(7), pp. 914-916.

8. Ramachandran M, Birch R, Eastwood DM. Clinical outcome of nerve injuries associated with supracondylar fractures of the humerus in children. The experience of a specialist referral centre. $J$ Bone Joint Surg Br. 2006, 88(1), pp. 90-94.

9. Oliver Eberhardt, Francisco Fernandez, Thomas Ilchmann \& Klaus Parsch. Cross pinning of supracondylar fractures from a lateral approach. Stabilization achieved with safety. J Child Orthop. 2007, 1, pp. 127-133.

10. Flynn, J.C. and Zink, W.P.: In: MacEwen GD, Kasser JR,Heinrich SD, eds. Pediatric fractures. A practical approach to assessment and treatment. Baltimore: Williams and Wilkins. 1993, pp. 133-164.

11. Brauer CA, Lee BM, Bae DS, Waters PM, Kocher MS.A. systematic review of medial and lateral entry pinning versus lateral entry pinning for supracondylar fractures of the humerus. J Pediatr Orthop. 2008, Vol. 2, 27, pp. 181-186.

12. Eberhardt O, Fernandez F, Ilchmann T, Parsch K. Cross pinning of supracondylar fractures from a lateral approach. Stabilization achieved with safety. $J$ Child Orthop. 2007, 1, pp. 127-133.

13. Gangadharan S, Rathinam B, Madhuri V. Radial nerve safety in Dorgan's lateral cross-pinning of the supracondylar. $J$ Pediatr Orthop B. 2014, 6, pp. 579-583.

14. Queally JM, Paramanathan N, Walsh JC, Moran CJ, Shannon FJ, D'Souza
LG. Dorgan's lateral cross-wiring of supracondylar fractures of the humerus in children: A retrospective review. $j$. injury. 2010, 6 .

15. Shannon FJ, Mohan P, Chacko J, D'Souza LG. Dorgan's percutaneous lateral cross wiring of supracondylar fractures of the humerus in children. $J$ Pediatr Orthop. 2004, 24, pp. 376-379.

16. Lee SS, Mahar AT, Miesen D, Newton PO. Displaced pediatric supracondylar humerus fractures: biomechanical analysis of percutaneous pinning techniques. $J$ Pediatr Orthop. 2002, 22, pp. 440-443.

17. Sudeep Vaidya, Achyut Rajbhandari, Nabees Pradhan, Suman Shrestha. Percutenious Fixation Of Displaced Supracondylar Fracture In Children Comparing Lateral With Medial And Lateral Pin. J Pediatr Orthop. 2009, 13, pp. 521-555.

18. El-Adl WA, El-Said MA, Boghdady GW, Ali AM. Results of treatment of displaced supracondylar humeral fractures in children by percutaneous lateral crosswiring technique. Strategies Trauma Limb Reconstr. 2008, Vol. 3, pp. 1-7.

19. Brown IC, Zinar DM. Traumatic and iatrogenic neurological complications after supracondylar fractures of the humerus in children. J Pediatr Orthop. 1995, 15, pp. 440-443.

20. Queally JM, Paramanathan N, Walsh JC, Cathal J Moran CJ, Fintan J, et al. Dorgan's lateral cross-wiring of supracondylar fractures of the humerus in children: a retrospective review. Injury. 2010, 41, pp. 568-571.

21. Flynn JC, Matthews JG, Benoit RL. Blind pinning of displaced supracondylar fractures of the humerus in children. Sixteen years' experience with long-term follow-up. J Bone Joint Surg Am. 1974, Vol. 2, 56, pp. 263-272. 